\title{
Aharonov-Bohm and Relativistic Corbino Effects in Graphene: A Comparative Study of Two Quantum Interference Phenomena
}

\author{
A. RYCERZ* \\ Instytut Fizyki im. Mariana Smoluchowskiego, Uniwersytet Jagielloński \\ W.S. Reymonta 4, PL-30-059 Kraków, Poland
}

\begin{abstract}
This is an analytical study of magnetic fields effects on the conductance, the shot noise power, and the third charge-transfer cumulant for the Aharonov-Bohm rings and the Corbino disks in graphene. The two distinct physical mechanisms lead to very similar magnetotransport behaviors. Differences are unveiled when discussing the third-cumulant dependence on magnetic fields.
\end{abstract}

PACS: 72.80.Vp, 73.43.Qt, 73.63.-b

\section{Introduction}

The advent of graphene, two-dimensional form of carbon in which itinerant electrons behave as massless Dirac fermions [1, 2], led condensed-matter physicists to reexamine effects of quantum transport in nanostructures [3]. The Aharonov-Bohm effect (ABE) [4], a famous condensed-matter realization of the two-slit gedankenexperiment [5], has also gained some attention [6-8]. Very recently, it was predicted theoretically [9] that periodic (approximately sinusoidal) magnetoconductance oscillations appear in weakly doped Corbino disks in graphene. Unlike ABE, which also appears for the Schrödinger electrons in the two-dimensional electron gas (2DEG), the quantum relativistic Corbino effect (QRCE) is a quantum-interference phenomenon specific for massless Dirac fermions, for which transmission via evanescent waves leads to a finite value of the conductance at zero doping.

It was also found in Refs. [9] and [10] that for QRCE in disks of moderate radii ratios $r_{2} / r_{1} \lesssim 10$ (see Fig. 1 ) two basic transport characteristics: the conductance $G$ and the shot-noise power (quantified by the Fano factor $F$ ) show qualitatively similar behavior as their ABE counterparts. In this paper we extent the discussion on the third charge-transfer cumulant [11] showing that this quantity (analyzed as a function of applied magnetic field) demonstrates several new features of QRCE absent in ABE, including the oscillations frequency doubling at $r_{2} / r_{1} \approx 7$. But first, we briefly recall the basic definitions of mesoscopic electron transport characteristics.

\footnotetext{
* e-mail: rycerz@th.if.uj.edu.pl
}

(a)
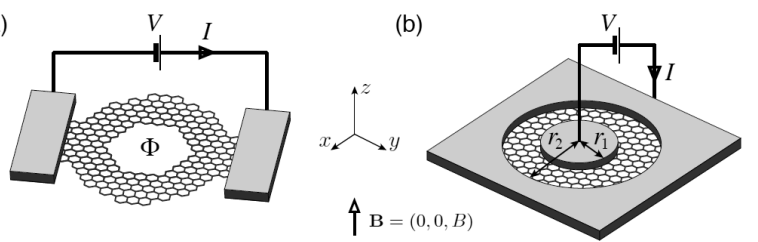

Fig. 1. Devices considered in the paper (schematic). Voltage source passes the current from the right to the left lead in case of the Aharonov-Bohm ring (a) or from the outer circular lead to the inner one in case of the Corbino disk (b) in graphene. The coordinate system and the applied magnetic field orientation (the same for both devices) are also depicted. Additional gate electrodes (not shown) may be used to tune dopings or to induce transverse electric fields.

\section{Counting statistics and charge cumulants}

Electric charge $Q$ passing the nanoscale device at the time interval $\Delta t$ in the presence of external bias voltage $V$ is a random variable, a statistical distribution of which can be expressed in terms of characteristic function

$$
\Lambda(\chi)=\langle\exp (\mathrm{i} \chi Q / e)\rangle,
$$

where the electron charge is $-e$ and $\langle X\rangle$ denotes the expectation value of $X$. In the so-called shot-noise limit $e V \gg k_{\mathrm{B}} T$ (with the Boltzmann constant $k_{\mathrm{B}}$ and temperature $T$ ) the characteristic function is given by [3]:

$$
\ln \Lambda(\chi)=\frac{s e V \Delta t}{2 \pi \hbar} \sum_{p} \ln \left(1+T_{p}\left(\mathrm{e}^{\mathrm{i} \chi}-1\right)\right),
$$

where $T_{p}$ are transmission probabilities for normal modes in leads, each of which has a degeneracy $s$ (at low fields, $s=2$ for $2 \mathrm{DEG}$, or $s=4$ for bulk graphene due to spin 
and valley degeneracy). We have assumed $V>0$ for simplicity; $\hbar$ denotes the Planck constant.

The average charge $\langle Q\rangle$, as well as higher charge-cumulants $\left\langle\left\langle Q^{k}\right\rangle\right\rangle \equiv\left\langle(Q-\langle Q\rangle)^{k}\right\rangle$ may be obtained by subsequent differentiation of $\ln \Lambda(\chi)$ with respect to i $\chi$ and setting $\chi=0$. In particular, the conductance

$$
G \equiv \frac{\langle Q\rangle}{V \Delta t}=\left.\frac{e}{V \Delta t} \frac{\partial \ln \Lambda}{\partial(\mathrm{i} \chi)}\right|_{\chi=0}=\frac{s e^{2}}{2 \pi \hbar} \sum_{p} T_{p},
$$

which restores the Landauer-Buttiker formula. Analogously, the Fano factor

$$
F \equiv \frac{\left\langle\left\langle Q^{2}\right\rangle\right\rangle}{\left\langle\left\langle Q^{2}\right\rangle\right\rangle_{\text {Poisson }}}=\frac{\sum_{p} T_{p}\left(1-T_{p}\right)}{\sum_{p} T_{p}},
$$

where $\left\langle\left\langle Q^{2}\right\rangle\right\rangle_{\text {Poisson }}=e\langle Q\rangle$ denotes the value of $\left\langle\left\langle Q^{2}\right\rangle\right\rangle$ for the Poissonian limit $T_{p} \ll 1$. We further define the $R$-factor, quantifying the third cumulant

$$
R \equiv \frac{\left\langle\left\langle Q^{3}\right\rangle\right\rangle}{\left\langle\left\langle Q^{3}\right\rangle\right\rangle_{\text {Poisson }}}=\frac{\sum_{p} T_{p}\left(1-T_{p}\right)\left(1-2 T_{p}\right)}{\sum_{p} T_{p}},
$$

with $\left\langle\left\langle Q^{3}\right\rangle\right\rangle_{\text {Poisson }}=e^{2}\langle Q\rangle$. For undoped graphene samples, similarly as for diffusive wires, the distribution of transmission eigenvalues is $\rho_{\text {diff }}(T)=$ $2 G /\left(g_{0} T \sqrt{1-T}\right)$ [12], with the conductance quantum $g_{0} \equiv 2 e^{2} / \pi \hbar$, leading to $F=1 / 3$ and $R=1 / 15$.

\section{Aharonov-Bohm effect in graphene}

Recent experimental [7] and numerical [8] studies suggest that the magnetoconductance of the AharonovBohm rings in graphene behaves similarly as the magnetoconductance of two parallel tunnel junctions [13]. In particular, the oscillations magnitude $\Delta G \equiv G_{\max }-$ $G_{\min } \propto G_{\mathrm{av}} \ll g_{0}$ (with $G_{\max }, G_{\min }$, and $G_{\mathrm{av}}$ the maximal, the minimal and the average value of the conductance when varying flux piercing the ring area $\Phi$; see Fig. 1a).

Such observations allow us to regard electron transport through a narrow symmetric ring as dominated by a single mode $(p=0)$ with

$$
T_{0}=\Gamma \cos ^{2}\left(\frac{\gamma_{0}}{2}+\frac{\pi \Phi}{\Phi_{\mathrm{AB}}}\right),
$$

where $\Gamma \leqslant 1$ is the transmission probability for each of the ring arms [14], $\gamma_{0}$ is the total dynamic phase gained by an electron traveling around the ring at zero magnetic field (typically, $\gamma_{0}$ is controlled by the transverse electric field induced by gate electrodes not shown in Fig. 1a), and $\Phi_{\mathrm{AB}} \equiv 2 \pi \hbar / e$ is the flux quantum. We notice here that randomly-chosen $\gamma_{0}$ leads to the distribution of transmission probabilities $\rho_{\mathrm{AB}}(T)=1 / \pi \sqrt{T(\Gamma-T)}$, with $0 \leqslant T \leqslant \Gamma$. Subsequently, $G=g_{0} \Gamma / 2, F=1 / 4$, and $R=0$, which reproduce the results for a symmetric chaotic cavity [3]. Although we consider a simple model (6), the universality of chaotic cavity transport properties lets us believe that these results also hold true for real systems. So far, the experimental values of $F$ and $R$ for the Aharonov-Bohm rings in graphene are unavailable.

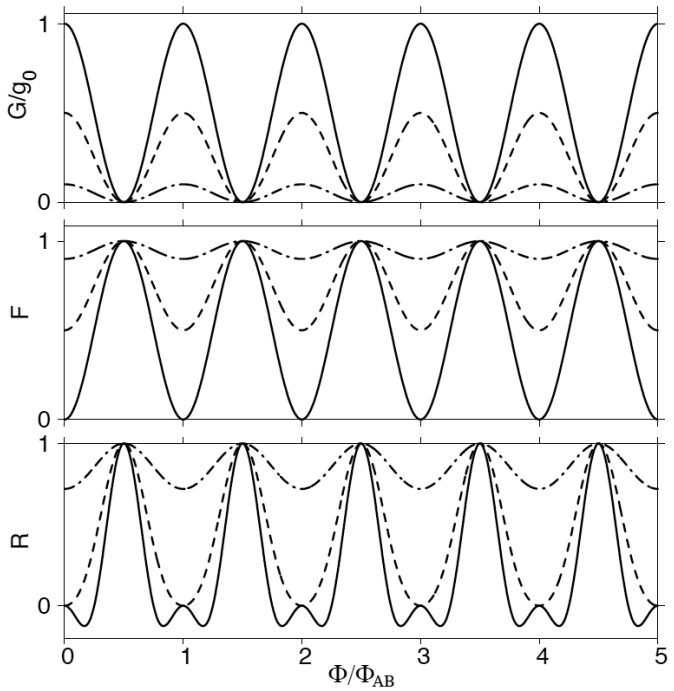

Fig. 2. Magnetic flux effect on the conductance (top part), the Fano factor (middle part) and the $R$-factor (bottom part) obtained from a single-mode model of transport via symmetric Aharonov-Bohm ring (6). The parameters are: $\Gamma=1$ (solid lines), $\Gamma=1 / 2$ (dashes lines), and $\Gamma=1 / 10$ (dashed-dot lines); $\gamma_{0}=0$ for all curves.

Substituting $T_{p}=T_{0} \delta_{0, p}$ into Eqs. (3)-(5) we obtain the conductance, the Fano factor, and the $R$-factor presented in Fig. 2 for $\gamma_{0}=0$ and selected values of $\Gamma$. Each of the studied quantities oscillates as a function of flux with period $\Phi_{\mathrm{AB}}$. The influence of higher harmonic frequencies becomes visible only for the $R$-factor if $\Gamma \gtrsim 1 / 2$. The first-harmonic amplitude, however, dominates the magnetic field dependence of $R$ for any $\Gamma$, and no qualitative effect (such as the frequency doubling) is present. Also, the oscillations magnitudes: $\Delta G / g_{0}=\Delta F=\Gamma$ and $\Delta R=\Gamma^{\prime}\left(3-2 \Gamma^{\prime}\right)$, with $\Gamma^{\prime}=\min \left(\Gamma, \frac{3}{4}\right)$, are all monotonically increasing functions of $\Gamma$.

\section{Relativistic Corbino effect}

Transmission probabilities for the Corbino disk in undoped graphene (see Fig. 1b) are given by [9]:

$$
T_{j}=\frac{1}{\cosh ^{2}\left[\left(j+\Phi_{12} / \Phi_{0}\right) \ln \left(r_{2} / r_{1}\right)\right]},
$$

where $j= \pm \frac{1}{2}, \pm \frac{3}{2}, \ldots$ is the angular momentum quantum number, $\Phi_{12}=\pi B\left(r_{2}^{2}-r_{1}^{2}\right)$ is the flux piercing the disk in the uniform magnetic field $B$ [15], and $\Phi_{0} \equiv$ $2 \Phi_{\mathrm{AB}} \ln \left(r_{2} / r_{1}\right)$ is the oscillation period of the conductance and higher charge-cumulants. Equation (7) holds true also in the small vicinity of the charge-neutrality point, defined via $\left|\Phi_{12}\right| \lesssim 2 \Phi_{\mathrm{AB}} \ln \left(\hbar v_{\mathrm{F}} /|\mu| r_{1}\right)$, where $v_{\mathrm{F}} \approx 10^{6} \mathrm{~m} / \mathrm{s}$ is the Fermi velocity in graphene and $\mu$ is the electrochemical potential. 


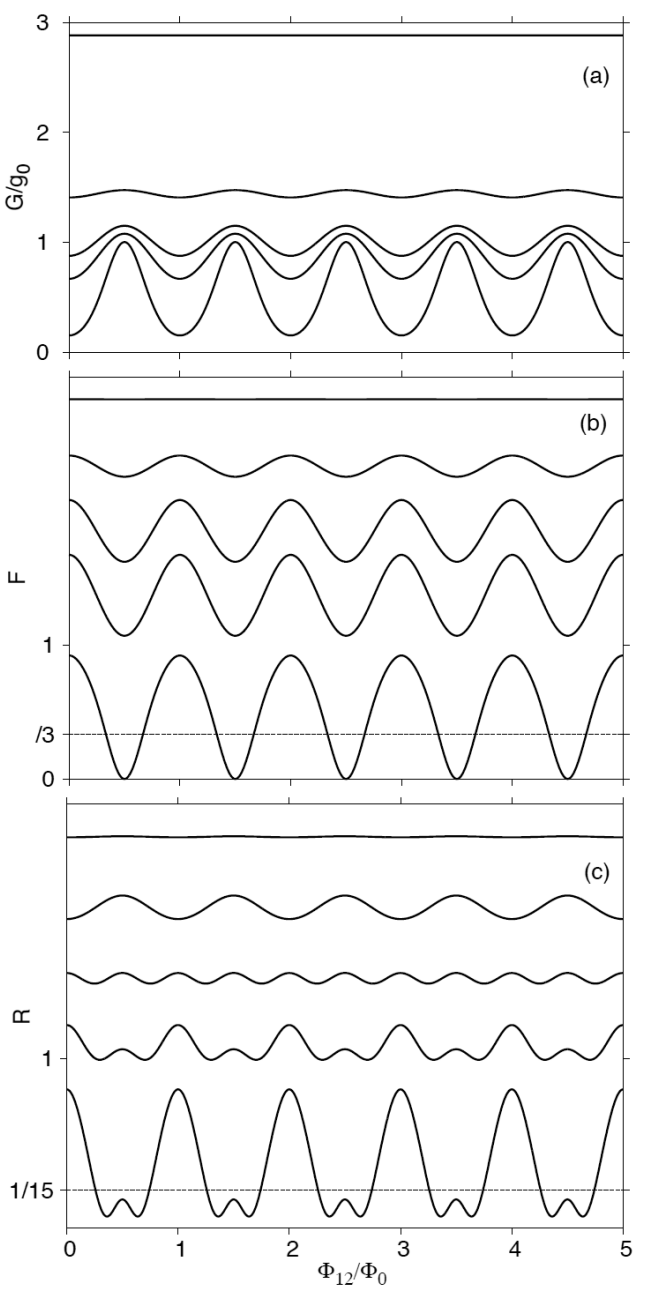

Fig. 3. The same as in Fig. 2 but for the undoped Corbino disks of radii ratios $r_{2} / r_{1}=2,4,7.2,10$, and 50 (top to bottom solid line at each part). In parts (b) and (c) all but the uttermost bottom curves are shifted upward for clarity. Diffusive values of $F$ and $R$ are also shown (dashed lines).

We now substitute $T_{j}$ to Eqs. (3)-(5) for measurable quantities. Results are presented in Fig. 3. For small values of $r_{2} / r_{1}$ we have $G \approx 2 g_{0} / \ln \left(r_{2} / r_{1}\right), F \approx 1 / 3$, and $R \approx 1 / 15$, reproducing the diffusive values. This is also worth to notice that taking random fluxes $\Phi_{12}$ covering uniformly the period $\Phi_{0}$, one restores $\rho_{\text {diff }}(T)$ for arbitrary $j$ and $r_{2} / r_{1}$ in Eq. (7). Subsequently, diffusive values of $G$ and higher charge cumulants $\left\langle\left\langle Q^{k}\right\rangle\right\rangle$ are exactly restored by averaging over magnetic fields for any $r_{2} / r_{1}$. For large $r_{2} / r_{1}$, the system alternates between two transport regimes when varying magnetic field: At $\Phi_{12} / \Phi_{0}$ close to half-odd integer, the transport is governed by a mode with $T_{j} \approx 1$, leading to $G \approx g_{0}, F \approx R \approx 0$, such as for a single-mode quantum point contact. On the contrary, when $\Phi_{12} / \Phi_{0}$ is close to an integer, we have $T_{j} \ll 1$ for all $j$-s, leading to $G \ll g_{0}, F \approx R \approx 1$, such as for a tunneling junction. This feature makes the system be- havior similar to that characteristic for a double barrier rather than the Aharonov-Bohm ring [3].

\section{Fourier analysis}

A deeper insight into the magnetic fields effect on transport via Corbino setup is provided with the Fourier decomposition of the measurable quantities. The following expressions are obtained employing the Poisson theorem:

$$
\begin{aligned}
& G=G_{0}\left(1+2 \sum_{n \geq 1} \alpha_{n} \cos \phi_{n}\right) \\
& F=\frac{\frac{1}{3}+2 \sum_{n \geq 1} \alpha_{n}\left(\frac{1}{3}-\frac{2 \pi^{2}}{3} l_{n}^{2}\right) \cos \phi_{n}}{1+2 \sum_{n \geq 1} \alpha_{n} \cos \phi_{n}}, \\
& R=\frac{\frac{1}{15}+2 \sum_{n \geq 1} \alpha_{n}\left(\frac{1}{15}-\frac{2 \pi^{2}}{3} l_{n}^{2}+\frac{4 \pi^{4}}{15} l_{n}^{4}\right) \cos \phi_{n}}{1+2 \sum_{n \geq 1} \alpha_{n} \cos \phi_{n}},
\end{aligned}
$$

with $G_{0} \equiv 2 g_{0} / \ln \left(r_{2} / r_{1}\right), \phi_{n} \equiv 2 \pi n \Phi_{12} / \Phi_{0}, l_{n} \equiv$ $n / \ln \left(r_{2} / r_{1}\right)$, and $\alpha_{n} \equiv(-1)^{n} \pi^{2} l_{n} / \sinh \left(\pi^{2} l_{n}\right)$.

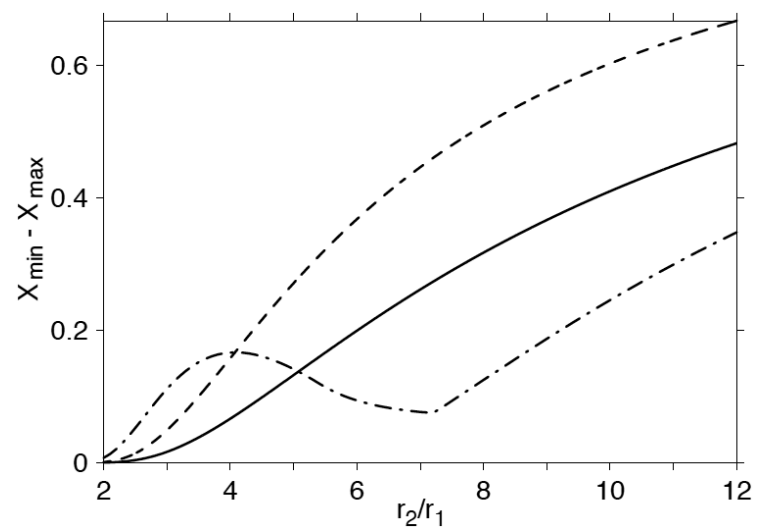

Fig. 4. Oscillations magnitude $X_{\max }-X_{\min }$ for $X=$ $G / g_{0}$ (solid line), $X=F$ (dashed line), and $X=R$ (dashed-dot line) as a function of the Corbino disk radii ratio $r_{2} / r_{1}$.

It is clear from Eqs. (8)-(10) that for the moderate values of $r_{2} / r_{1}$ (corresponding to large $l_{n}$-s) the oscillations magnitude of first three charge-cumulants grows systematically with the cumulant rank (see Fig. 4). In fact, $\Delta X \equiv X_{\max }-X_{\min }$ exceed $10 \%$ of the diffusive value for $r_{2} / r_{1}>4.9$ if $X=G / g_{0}$, for $r_{2} / r_{1}>2.8$ if $X=F$, and for $r_{2} / r_{1}>2.0$ if $X=R$. The behavior of charge cumulants for larger $r_{2} / r_{1}$ is, however, more complicated. Expanding Eqs. (8)-(10) up to the terms $\propto\left|\alpha_{1}\right|$ gives us

$$
\begin{aligned}
& G \approx G_{0}\left[1-2\left|\alpha_{1}\right| \cos \left(2 \pi \Phi_{12} / \Phi_{0}\right)\right], \\
& F \approx \frac{1}{3}+\frac{4 \pi^{2}}{3}\left|\alpha_{1}\right| l_{1}^{2} \cos \left(2 \pi \Phi_{12} / \Phi_{0}\right),
\end{aligned}
$$




$$
R \approx \frac{1}{15}+\frac{4 \pi^{2}}{3}\left|\alpha_{1}\right|\left(l_{1}^{2}-\frac{2 \pi^{2}}{5} l_{1}^{4}\right) \cos \left(2 \pi \frac{\Phi_{12}}{\Phi_{0}}\right) .
$$

Due to a fast decay of $\left|\alpha_{n}\right|$ with growing $n$, the above provides excellent approximations of $G$ and $F$ for $r_{2} / r_{1} \lesssim 10$. This is not the case for $R$, a first-harmonics amplitude of which changes the sign at $r_{2} / r_{1}=\exp (\sqrt{2 / 5} \pi) \approx$ 7.2 , leading to the oscillations frequency doubling (see Fig. 3c) and to a cusp-shaped local minimum on the $\Delta R$ plot (dashed-dot line in Fig. 4, notice also a local maximum at $r_{2} / r_{1} \approx 4$ ). For larger $r_{2} / r_{1}$-s, the magnitudes growths slowly approach the limiting value $\Delta G / g_{0}=\Delta F=\Delta R=1$, keeping the relation $\Delta R<$ $\Delta G / g_{0}<\Delta F$.

\section{Conclusions}

We find the third cumulant of charge transfer via the Corbino magnetometer in graphene with moderate (and thus most likely experimentally accessible) outer to inner radii ratios $r_{2} / r_{1}$ exhibits remarkably stronger oscillations with the varying field than earlier predicted for the conductance [9] and the shot-noise power [10]. The oscillations magnitude shows surprising features when studied as a function of $r_{2} / r_{1}$, including the maximum at $r_{2} / r_{1} \approx 4$, and cusp-shaped minimum accompanied by frequency doubling near $r_{2} / r_{1} \approx 7$. Such features, together with the size-dependent oscillations period, may help determining the effective proportions of ballistic graphene samples attached to metallic leads, at least in the case of a rotationally-symmetric setup.

Additionally, third cumulant appears to be the lowest in rank that is capable of illustrating the qualitative difference between electron magnetotransport through the Aharonov-Bohm and the Corbino quantum interference devices in graphene. Certain features of such two systems suggest that the former and the latter may reproduce transport properties of chaotic cavities and diffusive wires (respectively).

\section{Acknowledgments}

The work was supported by the National Science Centre of Poland (NCN) via grant No. N-N202-031440, by the Alexander von Humboldt Stiftung-Foundation, and partly by Foundation for Polish Science (FNP) under the program TEAM.

\section{References}

[1] G.W. Semenoff, Phys. Rev. Lett. 53, 2449 (1984).

[2] K.S. Novoselov, A.K. Geim, S.V. Morozov, D. Jiang, M.I. Katsnelson, I.V. Grigorieva, S.V. Dubonos, A.A. Firsov, Y. Zhang, Nature 438, 197 (2005).

[3] Yu.V. Nazarov, Ya.M. Blanter, Quantum Transport: Introduction to Nanoscience, Cambridge University Press, Cambridge 2009.

[4] Y. Imry, R.A. Webb, Sci. Am. 260, 36 (1989).

[5] K. Popper, The Logic of Scientific Discovery, Routledge Classics, New York 2002, Appendix V.

[6] P. Recher, B. Trauzettel, A. Rycerz, Ya.M. Blanter, C.W.J. Beenakker, A.F. Morpurgo, Phys. Rev. B 76, 235404 (2007); M. Zarenia, J.M. Pereira Jr., A. Chaves, F.M. Peeters, G.A. Farias, ibid., 81, 045431 (2010).

[7] S. Russo, J.B. Oostinga, D. Wehenkel, H.B. Heersche, S.S. Sobhani, L.M.K. Vandersypen, A.F. Morpurgo, Phys. Rev. B 77, 085413 (2008); C. Stampfer, E. Schurtenberger, F. Molitor, J. Guettinger, T. Ihn, K. Ensslin, Int. J. Mod. Phys. 23, 2647 (2009).

[8] A. Rycerz, Acta Phys. Pol. A 115, 322 (2009); J. Wurm, M. Wimmer, H.U. Baranger, K. Richter, Semicond. Sci. Technol. 25, 034003 (2010); J. Schelter, D. Bohr, B. Trauzettel, Phys. Rev. B 81, 195441 (2010).

[9] A. Rycerz, Phys. Rev. B 81, 121404(R) (2010).

[10] M.I. Katsnelson, Europhys. Lett. 89, 17001 (2010); M.I. Katsnelson, J. Comput. Theor. Nanosci. 8, 912 (2011).

[11] B. Reulet, J. Senzier, D.E. Prober, Phys. Rev. Lett. 91, 196601 (2003); Y. Bomze, G. Gershon, D. Shovkun, L.S. Levitov, M. Reznikov, Phys. Rev. Lett. 95, 176601 (2005).

[12] C.W.J. Beenakker, Rev. Mod. Phys. 80, 1337 (2008).

[13] Yu.V. Nazarov, Phys. Rev. B 47, 2768 (1993).

[14] Typically, $\Gamma \ll 1$ as for graphene rings with irregular edges there are only evenascent modes present in each of the ring arms at zero and weak dopings, see Refs. $[7,8]$.

[15] More generally, for centrosymmetric field $\boldsymbol{B}=$ $B(r) \hat{e}_{z}$, the parameter $\phi=\Phi_{12} / \Phi_{0}$ in Eq. (7) is replaced by [10]: $\phi^{\prime} \equiv \frac{\Phi_{1}}{\Phi_{\mathrm{AB}}}+\frac{4 \pi}{\Phi_{0}} \int_{r_{1}}^{r_{2}} \frac{\mathrm{d} r}{r} \int_{r_{1}}^{r} \mathrm{~d} r^{\prime} r^{\prime} B(r)$, where $\Phi_{1}$ is flux through the inner ring $\left(r<r_{1}\right)$. In particular, if $B(r)=0$ for $r>r_{1}$, the AharonovBohm-like oscillations occur when varying $\Phi_{1}$. 